\title{
The Entropy of Supermassive Black Holes during Its Evaporation Time ${ }^{\dagger}$
}

\section{R. Leticia Corral-Bustamante}

Instituto Tecnológico de Cd. Cuauhtémoc, Cuauhtémoc, Chihuahua, Mexico

+ Presented at the Entropy 2021: The Scientific Tool of the 21st Century, 5-7 May 2021; Available online: https://sciforum.net/conference/Entropy2021/.

Published: 5 May 2021

Is it possible to quantify in General Relativity, $R G$, the entropy generated by supermassive black holes, BHs, during its evaporation time, since the intrinsic Hawking radiation in the infinity that, although insignificant, is important in the effects on the thermal quantum atmosphere?

The purpose was to develop a formula that allows us to measure the entropy generated during the evaporation time of different types of BHs of: i. remnant BH of the binary black holes' merger, $\mathrm{BBH}$ : GW150914, GW151226 and LTV151012 detected by the Laser Interferometer Gravitational-Wave Observatory (LIGO), and ii. Schwarzschild, Reissner-Nordström, Kerr and Kerr-Newman, and thus quantify in GR the "insignificant" quantum effects involved, in order to contribute to the validity of the generalized second law (GSL) that directly links the laws of black hole mechanics to the ordinary laws of thermodynamics, as a starting point for unifying quantum effects with GR. This formula could have some relationship with the detection of the shadow's image of the event horizon of a BH.

This formula was developed in dimensional analysis, using the constants of nature and the possible evaporation time of a black hole taking into account its distance to the Earth, to quantify the entropy generated during that time. The energy-stress tensor was calculated with the 4 metrics to obtain the material content and apply the proposed formula.

The entropy of the evaporation time of BHs proved to be insignificant, its temperature is barely above absolute zero, however, the calculation of this type of entropy allows us to argue about the importance of the quantum effects of Hawking radiation mentioned by authors who have studied the quantum effects with arguments that are fundamentally based on the presence of the surrounding thermal atmosphere of the black hole.

(C) 2021 by the author. Licensee MDPI, Basel, Switzerland. This article is an open access article distributed under the terms and conditions of the Creative Commons Attribution (CC BY) license (http://creativecommons.org/licenses/by/4.0/). 